9. D. Ray, On spectra of second order differential operators, Trans. Amer. Math. Soc. 77 (1954), 299-321.

10. F. Riesz and B. Sz.-Nagy, Functional analysis, trans. from 2nd French edition by L. Boron, Ungar, New York, 1955.

11. F. Rellich, Perturbation theory of eigenvalue problems, Lecture Notes, New York University, New York, 1955.

12. E. C. Titchmarsh, Eigenfunction expansions associated with second-order differential equations, Part II, Oxford Univ. Press, New York, 1958.

University of California, Los Angeles

\title{
DISTRIBUTION MODULO 1 AND SETS OF UNIQUENESS
}

\author{
BY J.-P. KAHANE AND R. SALEM $\dagger$
}

Communicated by A. Zygmund, July 6, 1963

A linear set $E \subset(0,1)$ is said to be a set of uniqueness (set $U$ ) for trigonometric expansion if no trigonometric series exists (except vanishing identically) which converges to zero in the set $C E$ complementary to $E$. Following Nina Bary we shall say that $E$ is a set of uniqueness "in the wide sense" (set $U^{*}$ ) if no Fourier-Stieltjes series exists (except vanishing identically) which converges to zero in $C E$. If $E$ is a closed set $U^{*}$ it means (see [1, Vol. 1, pp. 344-359, Vol. 2, p. 160]) that $E$ does not carry any measure whose Fourier-Stieltjes coefficients tend to zero. If $E$ is a closed set $U$ (i.e. of uniqueness "strict sense") it means that $E$ does not carry any measure or pseudo-measure (cf. [2]) with coefficients tending to zero.

Definition. A real sequence of numbers $\left\{u_{\kappa}\right\}_{1}^{\infty}$ will be said to be "badly distributed" modulo 1 if there exists at least one characteristic function $X(x)$ of open interval $\Delta \subset(0,1)$ periodic with period 1 such that

$$
\limsup _{\kappa=\infty} \frac{X\left(u_{1}\right)+\cdots+X\left(u_{\kappa}\right)}{\kappa}<\int_{0}^{1} X(x) d x=|\Delta|
$$

when $|\Delta|$ stands for the length of $\Delta .{ }^{1}$

REMARK. It is easy to see that under this hypothesis there exists a $\Delta$ with rational end-points having the same property.

THEOREM. Let $E \subset(0,1)$ be a linear set such that there exists an infinite sequence of positive integers $\left\{n_{\kappa}\right\}_{1}^{\infty}$ increasing to infinity, with the

$\dagger$ Professor Salem died June 20, 1963, in Paris.

1 The reader will convince himself that all the argument which follows is applicable in the case we suppose $\lim$ inf $>\Delta$. 
property that for every $x \in E$, the sequence $\left\{n_{\kappa} x\right\}$ is badly distributed modulo 1 . Then $E$ is a set of the type $U^{*}$.

We shall make use of the three following known lemmas:

Lemma I (see $[1$, Vol. 2, pp. 145, 160]). In order to prove that a closed set does not carry a nonvanishing measure with Fourier-Stieltjes coefficients tending to zero, it is sufficient to prove that it does not carry a positive measure having this property.

Lemma II (see [1, Vol. 2, p. 144]). Let

$$
d \mu \sim \sum_{-\infty}^{\infty} c_{n} e^{2 \pi i n x}
$$

be a Fourier-Stieltjes series and let

$$
X(x) \sim \sum_{-\infty}^{\infty} \gamma_{n} e^{2 \pi i n x}
$$

be the Fourier series of the characteristic function $X(x)$ of an interval $\Delta \subset(0,1)$. Then if the Fourier-Stieltjes coefficients $c_{n}$ tend to zero as $n \rightarrow \infty$, one has

$$
\lim _{m=\infty} \int_{0}^{1} X(m x) d \mu=c_{0} \gamma_{0}=\int_{0}^{1} X(x) d x \cdot \int_{0}^{1} d \mu(x) .
$$

Lemma III (see [1, Vol. 2, p. 160]). A set $E$ which is the union of a denumerable infinity of closed sets $F_{n}$ each of which is of the type $U^{*}$, is also of the type $U^{*}$.

ProOF OF THE THEOREM. Taking into account the remark following the definition of "bad distribution," we see that to every $x \in E$ corresponds a characteristic function of open interval with rational endpoints, thus belonging to a denumerable family $\left\{X_{m}(x)\right\}_{1}^{\infty}$. Let $E_{m}$ be the subset of points $x$ of $E$ corresponding to the same function $X_{m}(x)$. $E$ is the union of all the sets $E_{m}$.

The set $E_{m}$ is itself the union of sets $E_{m, h}(h$ a positive integer $1 \leqq h<\infty)$ such that

$$
\frac{X_{m}\left(n_{1} x\right)+\cdots+X_{m}\left(n_{k} x\right)}{\kappa}<\int_{0}^{1} X_{m}(x) d x \text { for } \quad \kappa \geqq h .
$$

The set $E_{m, h}$ is in turn the union of sets $E_{m, h, s}$, where

$$
\frac{X_{m}\left(n_{1} x\right)+\cdots+X_{m}\left(n_{k} x\right)}{\kappa} \leqq \int_{0}^{1} X_{m}(x) d x-\frac{1}{s} \quad(\kappa \geqq h)
$$

where $s$ takes all positive integral values. 
Since $X_{m}(x)$ is lower-semicontinuous, the set $E_{m, h, s}$ is closed. We shall show that it is of the type $U^{*}$. Suppose, in fact, that it carries a positive (see Lemma I) nonvanishing measure $d \mu$ with Fourier-Stieltjes coefficients tending to zero. Multiplying (1) by $d \mu$ and integrating with respect to $d \mu$ we set

$$
\underline{\int X_{m}\left(n_{1} x\right) d \mu+\cdots+\int X_{m}\left(n_{\kappa} x\right) d \mu}
$$

$$
\kappa
$$

$$
\leqq\left(\int_{0}^{1} X_{m} d x\right)\left(\int_{0}^{1} d \mu(x)\right)-\frac{\int_{0}^{1} d \mu}{s} \quad(\kappa \geqq h) .
$$

Since for $\kappa \rightarrow \infty$ the first member tends (Lemma II) to $\int U_{m} d x \cdot \int_{0}^{1} d \mu(x)$, this leads to a contradiction, and $E_{m, h, s}$ is a $U^{*}$ set.

It is now enough to use Lemma III to prove the theorem since $E$ is the union of the denumerable family $E_{m, h, s}(m, h, s$ positive integers).

Application. Consider the set of numbers in $(0,1)$ written in the dyadic system $x=\epsilon_{1} / 2+\cdots+\epsilon_{\kappa} / 2^{\kappa}+\cdots\left(\epsilon_{\kappa}=0,1\right)$ having the property that

$$
\lim \sup \frac{\epsilon_{1}+\cdots+\epsilon_{\kappa}}{\kappa}<\frac{1}{2} .
$$

This set is of the type $U^{*}$. This is an immediate consequence of our theorem if we remark that $\epsilon_{\kappa}=X\left(2^{*} x\right)$ when $X(x)$ is the characteristic function of the interval $(1 / 2,1)$. (Here the family $X_{m}$ is reduced to a single interval. ${ }^{2}$ )

\section{BIBLIOGRAPHY}

1. A. Zygmund, Trigonometric series, 2nd ed., Cambridge Univ. Press, 1959.

2. J.-P. Kahane and R. Salem, Sur les ensembles linéaires ne portant pas de pseudomesures, C. R. Acad. Sci. Paris 243 (1956), 1185-1187.

\section{UnIVERSity of Paris}

${ }^{2}$ Added in proof. The application given here is nothing but the easy part of an important paper of I. I. Pyatetski-Shapiro (Moskov. Gos. Univ. Uc. Zap. 165, Mathematika 7 (1954), 79-97), where he constructs a set $U^{*}$ which is not a set $U$. It suggests the following question, that the authors were not able to solve: is the set of nonnormal numbers $x$ (i.e., numbers $x$ such that $\lim \sup \left(\epsilon_{1}+\cdots+\epsilon_{K}\right) / K>1 / 2$ or $\lim \inf <1 / 2)$ a set $U^{*}$ ? In other words, is it a set of measure zero with respect to every positive measure whose Fourier coefficients tend to zero at infinity? 